\title{
The effect of nitroglycerin on hemodynamic changes during laparoscopic low anterior resection
}

\author{
Hyun Soo Moon ${ }^{1}$, Soo Kyung Lee ${ }^{1}$, Young Sil Choi ${ }^{1}$, Chi Bum $\operatorname{In}^{1}$, and Eun Joo Choi ${ }^{2}$ \\ Department of Anesthesiology and Pain Medicine, ${ }^{1}$ College of Medicine, Hallym University, Anyang, ${ }^{2}$ Seoul National University \\ Bundang Hospital, Seongnam, Korea
}

Background: More laparoscopic low anterior resections (LAR) are being performed in recent years. There has been controversy around the hemodynamic changes affected by the Trendelenburg position and pneumoperitoneum during LAR. The goal of this study was to analyze the effect of nitroglycerin (NTG) on hemodynamic changes during LAR.

Methods: Forty ASA physical status I-II patients undergoing LAR were randomized into two groups: the NTG infused group ( $\mathrm{N}$ group, $\mathrm{n}=20$ ) and the control group ( $\mathrm{C}$ group, $\mathrm{n}=20$ ). Anesthesia was maintained with sevoflurane at $1-3 \mathrm{vol} \%$, air/oxygen $(50 \% / 50 \%)$ and continuous infusion with remifentanil. The $\mathrm{N}$ group patients were given $0.5 \mu \mathrm{g} / \mathrm{kg} / \mathrm{min}$ of NTG during anesthesia. Mean arterial pressure (MAP), heart rate (HR), central venous pressure (CVP), cardiac index (CI), stroke volume (SV) and systemic vascular resistance (SVR) were assessed 10 min after induction (T1), 5 min after pneumoperitoneum in the supine position (T2), $10 \mathrm{~min}$ after pneumoperitoneum in the Trendelenburg position (T3), 30 min after pneumoperitoneum in the Trendelenburg position (T4), $1 \mathrm{hr}$ after pneumoperitoneum in the Trendelenburg position (T5) in addition to 5 (T6), 10 (T7) and $30 \mathrm{~min}$ (T8) after removal of the pneumoperitoneum in the supine position.

Results: The increases of MAP were milder in the N group (22.6-7.3\%) than the C group (32.3-17.7\%) during pneumoperitoneum and while in the Trendelenburg position. The significant decreases of HR were maintained in the $\mathrm{C}$ group, but the changes in HR were not significant in $\mathrm{N}$ group during the operation. The increases in CVP were less in $\mathrm{N}$ group than $\mathrm{C}$ group. The increases of SVR were milder in N group $(19.4-1.4 \%)$ than $\mathrm{C}$ group $(41.7-16.6 \%)$ during pneumoperitoneum in the Trendelenburg position.

Conclusions: Intraoperative NTG infusions were effective to some degree in reducing the hemodynamic changes during pneumoperitoneum with Trendelenburg positioning for LAR. (Korean J Anesthesiol 2011; 61: 388-393)

Key Words: Hemodynamic change, Nitroglycerin, Pneumoperitoneum, Trendelenburg position.

Received: February 28, 2011. Revised: 1st, May 12, 2011; 2nd, May 23, 2011. Accepted: May 23, 2011.

Corresponding author: Hyun Soo Moon, M.D., Department of Anesthesiology and Pain Medicine, Hallym University Sacred Heart Hospital, 896, Pyeongchon-dong, Dongan-gu, Anyang 431-070, Korea. Tel: 82-31-380-3945, Fax: 82-31-385-3244, E-mail: hysomoon@yahoo.co.kr (c) This is an open-access article distributed under the terms of the Creative Commons Attribution Non-Commercial License (http:// creativecommons.org/licenses/by-nc/3.0/), which permits unrestricted non-commercial use, distribution, and reproduction in any medium, provided the original work is properly cited. 


\section{Introduction}

Minimally invasive surgery using laparoscopy has become a widely used technique in various fields of general surgery, gynecology, and urology. The number of cases continue to increase because of its clinical advantages in decreasing perioperative complications, postoperative pain and postoperative hospitalization compared to the conventional open technique [1]. Pneumoperitoneum with carbon dioxide $\left(\mathrm{CO}_{2}\right)$ insufflation for visual fields can decrease thoracic and pulmonary compliances, atelectasis, pulmonary hypertension and hypercapnia due to increased abdominal pressure [2]. $\mathrm{CO}_{2}$ pneumoperitoneum also induces hemodynamic instabilities such as a decrease in cardiac output (CO), unstable blood pressure as well as increases in systemic and pulmonary vascular resistances [3]. Therefore, the risks during laparoscopic operations and anesthesia increase, especially in elderly or hemodynamically unstable patients.

For acquiring better visual fields and reducing blood loss during laparoscopic surgical procedures involving the lower abdomen including the colon, the Trendelenburg position has been used. This head-down positioning affects the pulmonary system by decreasing pulmonary compliance and the cardiovascular system by increasing preload and fluctuation of CO, thereby increasing pulmonary artery pressure and altering the variability of systemic vascular resistance (SVR) [4]. In a recent study [5], the increase in venous return by Trendelenburg positioning can compensate for the decrease in CO induced by pneumoperitoneum and this results in an insignificant change in net CO. However, the importance of careful anesthetic monitoring is essential as laparoscopic surgery of the colon requires a relatively long time which increases the risks associated with prolonged pneumoperitoneum and the Trendelenburg position. To minimize the hemodynamic and pathophysiologic influences induced by pneumoperitoneum and the Trendelenburg position during low anterior resection (LAR), proper maintenance of preload, pulmonary and systemic vascular resistance are required and agents including vasodilator, high dose opioids, calcium channel blockers and beta blockers can be selectively used. The purpose of this study was to evaluate the modulating effects of nitroglycerin on adverse hemodynamic changes induced by pneumoperitoneum and the Trendelenburg position during anesthesia for LAR.

\section{Materials and Methods}

Forty patients categorized as American Society of Anesthesiologists classification of physical status 1 or 2 , scheduled for elective LAR were enrolled in this study. All patients were informed of the methods and purpose of the study, and informed consents were obtained. This study was approved by the Institutional Review Board of our hospital. Patients with severe hypertension, hypotension, chronic obstructive pulmonary disease, cardiac diseases, morbid obesity (BMI > 30 ), cerebrovascular disease, severe liver or renal diseases in addition to having a history of psychiatric diseases were excluded from the study. Additionally, patients showing fluctuation in their blood pressure by $\pm 40 \%$ during LAR were also excluded from the analysis.

Patients were premedicated with $0.2 \mathrm{mg}$ of glycopyrrolate administered intramuscullary one hour before the induction of anesthesia. Upon arriving at the operation room, standard monitoring devices were applied including electrocardiogram, noninvasive blood pressure and pulse oximetry with a patient monitoring device (S/5, Datex-Ohmeda CO., UK). Induction of anesthesia was done with intravenous $1 \mu \mathrm{g} / \mathrm{kg}$ of remifentanil and $5 \mathrm{mg} / \mathrm{kg}$ of thiopental; level of sedation was monitored using the bispectral index (BIS: A-2000, Aspect Medical Co., USA). Endotracheal intubation was facilitated with intravenous rocuronium $(0.7 \mathrm{mg} / \mathrm{kg})$. After the induction of anesthesia, the patients' lungs were ventilated to maintain an end-tidal carbon dioxide between $33-37 \mathrm{mmHg}$ during the operation. Then a $20 \mathrm{G}$ arterial catheter was placed in the left radial artery to continuously monitor arterial blood pressure and to sample for intermittent arterial blood gas and a $7 \mathrm{~F}$ double lumen central venous catheter was placed at the right internal jugular vein to maintain fluid line and to monitor central venous pressure. An esophageal doppler (Cardio $\mathrm{Q}^{\mathrm{TM}}$, Deltex Medical Co., UK) was inserted for determining various hemodynamic parameters such as CO, stroke volume (SV), ejection fraction and SVR.

Anesthesia was maintained with 1-3 vol\% sevoflurane in air/oxygen $(2 \mathrm{~L} / 2 \mathrm{~L} / \mathrm{min})$ mixuture and continuous infusion of remifentanil (0.05-0.2 $\mu \mathrm{g} / \mathrm{kg} / \mathrm{min})$, keeping the BIS number between $40-60$. Rocuronium $(0.2-0.3 \mathrm{mg} / \mathrm{kg} / \mathrm{hr})$ was infused continuously for adequate muscle relaxation. Patients were randomly assigned into two groups in which the experimental group (nitroglycerin, $\mathrm{N}$ ) received $0.5 \mu \mathrm{g} / \mathrm{kg} / \mathrm{min}$ nitroglycerin from the beginning of anesthesia until the operation was finished, whereas the nitroglycerin was not given in the control group (C).

Intraoperative pneumoperitoneum was maintained at a pressure of 12-13 mmHg. Hartmann's solution was given at a rate of $2-4 \mathrm{ml} / \mathrm{kg} / \mathrm{hr}$ as the main fluid and packed RBC was given for blood loss during the operation to maintain preoperative hemoglobin values. Hemodynamic measurements including mean arterial pressure (MAP), heart rate (HR), central venous pressure (CVP), cardiac index (CI), stroke volume (SV) and systemic vascular resistance (SVR) were checked and recorded in both groups. Times of measurements were $10 \mathrm{~min}$ 
after induction of anesthesia (T1), 5 min after $\mathrm{CO}_{2}$ insufflation in the supine position (T2), 10 min after Trendelenburg positioning with pneumoperitoneum (T3), 30 min after Trendelenburg positioning with pneumoperitoneum (T4), 1 $\mathrm{hr}$ after Trendelenburg positioning with pneumoperitoneum (T5), 5 min after desufflation in the supine position (T6), $10 \mathrm{~min}$ after desufflation in the supine position (T7) and $30 \mathrm{~min}$ after desufflation in the supine position (T8), respectively.

All data were expressed as means \pm SD or number of patients. Intergroup data were analyzed using a Student $\mathrm{T}$ test and continuous data within groups were performed using repeated measures ANOVA followed by Bonferroni correction for multiple comparisons. $\mathrm{P}$ values $<0.05$ were regarded as statistically significant.

Table 1. Demographic Characteristics

\begin{tabular}{lcc}
\hline & $\mathrm{C}(\mathrm{n}=20)$ & $\mathrm{N}(\mathrm{n}=20)$ \\
\hline Age $(\mathrm{yr})$ & $62.8 \pm 13.3$ & $64.2 \pm 11.2$ \\
Gender $(\mathrm{M} / \mathrm{F})$ & $12 / 8$ & $13 / 7$ \\
Body weight $(\mathrm{kg})$ & $58.2 \pm 13.2$ & $59.3 \pm 13.8$ \\
Height $(\mathrm{cm})$ & $162.3 \pm 18.5$ & $164.5 \pm 16.6$ \\
\hline
\end{tabular}

Values are expressed as means \pm SD. C: control group, N: nitroglycerin group.

\section{Results}

Forty patients were included in this study and there were no significant differences between the control group (C) and the nitroglycerin group $(\mathrm{N})$ with respect to age, gender, body weight and height (Table 1).

MAPs were significantly increased in both groups at T2T4 during pneumoperitoneum compared to (T1) as well as in T5 of the C group. Ten min after Trendelenburg positioning with pneumoperitoneum (T3), there was a $32.3 \%$ increase in C group MAP which was higher than the $22.6 \%$ increase in the $\mathrm{N}$ group, although the difference was not significant between the two groups. HR significantly decreased at T3, T4 and $\mathrm{T} 5$ compared to $\mathrm{T} 1$ in the $\mathrm{C}$ group only and the $\mathrm{N}$ group had a significant increase at $\mathrm{T} 4$ when compared to the $\mathrm{C}$ group (Table 2). CVP increased significantly in both groups during the pneumoperitoneum (T2-T5) and when T6 was compared to $\mathrm{T} 1$, but there were no significant intergroup differences. CI was also decreased in both groups during the pneumoperitoneum (T2-T5) compared to $\mathrm{T} 1$ and significant decreases were noted at $\mathrm{T} 4$ and $\mathrm{T} 5$ which recovered after pneumoperitoneum was terminated: no differences were found between groups (Table 2 and 3). SV increased from T4 to T7 in C group while N group

Table 2. Hemodynamic Data during Laparoscopic Low Anterior Resection

\begin{tabular}{|c|c|c|c|c|c|c|c|c|c|}
\hline \multicolumn{2}{|c|}{ Parameter group } & $\mathrm{T} 1$ & $\mathrm{~T} 2$ & T3 & $\mathrm{T} 4$ & $\mathrm{~T} 5$ & T6 & $\mathrm{T} 7$ & T8 \\
\hline \multirow[t]{2}{*}{ MAP (mmHg) } & $\mathrm{C}$ & $92.3 \pm 12.5$ & $110.7 \pm 13.5^{*}$ & $122.1 \pm 15.2^{*}$ & $117.3 \pm 14.8^{*}$ & $108.6 \pm 14.2^{*}$ & $95.4 \pm 13.3$ & $90.8 \pm 13.5$ & $89.6 \pm 12.5$ \\
\hline & $\mathrm{N}$ & $91.7 \pm 13.8$ & $106.0 \pm 14.4^{*}$ & $112.4 \pm 14.5^{*}$ & $106.9 \pm 10.5^{*, \dagger}$ & $98.4 \pm 11.2$ & $92.3 \pm 10.5$ & $87.9 \pm 10.6$ & $87.6 \pm 12.3$ \\
\hline \multirow[t]{2}{*}{ HR (beats/min) } & $\mathrm{C}$ & $78.4 \pm 10.5$ & $69.2 \pm 11.2$ & $68.1 \pm 12.5^{*}$ & $67.3 \pm 11.8^{*}$ & $68.0 \pm 10.9 *$ & $70.0 \pm 10.0$ & $74.3 \pm 14.8$ & $76.5 \pm 15.2$ \\
\hline & $\mathrm{N}$ & $79.1 \pm 12.4$ & $76.4 \pm 10.7$ & $75.1 \pm 9.3^{\dagger}$ & $75.0 \pm 10.2^{\dagger}$ & $73.9 \pm 10.5$ & $72.4 \pm 11.8$ & $73.3 \pm 12.4$ & $74.9 \pm 12.8$ \\
\hline \multirow[t]{2}{*}{$\mathrm{CVP}\left(\mathrm{cmH}_{2} \mathrm{O}\right)$} & $\mathrm{C}$ & $5.0 \pm 1.6$ & $10.6 \pm 3.3^{*}$ & $11.9 \pm 2.8^{*}$ & $12.2 \pm 4.2^{*}$ & $11.9 \pm 3.4^{*}$ & $6.6 \pm 2.1^{*}$ & $5.6 \pm 2.4$ & $4.6 \pm 1.8$ \\
\hline & $\mathrm{N}$ & $4.6 \pm 2.0$ & $10.4 \pm 2.4^{*}$ & $11.6 \pm 3.3^{*}$ & $11.7 \pm 2.8 *$ & $10.8 \pm 3.3^{*}$ & $6.2 \pm 2.8^{*}$ & $4.8 \pm 2.0$ & $4.2 \pm 1.7$ \\
\hline
\end{tabular}

Values are expressed as means \pm SD. MAP: mean arterial pressure, HR: heart rate, CVP: central venous pressure, C: control group, N: nitroglycerin group, T1: $10 \mathrm{~min}$ after induction of anesthesia, T2: 5 min after $\mathrm{CO}_{2}$ insufflation in the supine position, T3: 10 min after the Trendelenburg position with pneumoperitoneum, T4: $30 \mathrm{~min}$ after the Trendelenburgposition with pneumoperitoneum, T5: 1 hr after the Trendelenburg position with pneumoperitoneum, T6: 5 min after desufflation in the supine position, T7: 10 min after desufflation in thesupine position, T8: 30 min after desufflation in the supine position. *Indicates $\mathrm{P}<0.05$ compared with $\mathrm{T} 1{ }^{\dagger}$ Indicates $\mathrm{P}<0.05$ compared with the same as the $\mathrm{C}$ group.

Table 3. Hemodynamic Data during Laparoscopic Low Anterior Resection

\begin{tabular}{|c|c|c|c|c|c|c|c|c|c|}
\hline Parameter grc & up & $\mathrm{T} 1$ & $\mathrm{~T} 2$ & T3 & $\mathrm{T} 4$ & T5 & $\mathrm{T} 6$ & $\mathrm{~T} 7$ & T8 \\
\hline \multirow[t]{2}{*}{$\mathrm{CI}\left(\mathrm{L} / \mathrm{min} / \mathrm{m}^{2}\right)$} & $\mathrm{C}$ & $3.0 \pm 0.8$ & $2.6 \pm 0.6$ & $2.7 \pm 0.8$ & $2.5 \pm 0.6^{*}$ & $2.5 \pm 0.8^{*}$ & $3.1 \pm 1.0$ & $3.2 \pm 0.9$ & $3.2 \pm 0.9$ \\
\hline & $\mathrm{N}$ & $2.9 \pm 1.2$ & $2.7 \pm 0.5$ & $2.8 \pm 0.6$ & $2.5 \pm 0.4^{*}$ & $2.6 \pm 0.7^{*}$ & $3.1 \pm 0.9$ & $3.1 \pm 1.0$ & $3.3 \pm 1.2$ \\
\hline \multirow[t]{2}{*}{$\mathrm{SV}$ (ml/beat) } & $\mathrm{C}$ & $57.6 \pm 10.8$ & $54.6 \pm 11.2$ & $61.3 \pm 12.3$ & $64.3 \pm 9.8^{*, \neq}$ & $64.2 \pm 13.5^{*, \neq}$ & $64.0 \pm 15.1^{*}$ & $64.5 \pm 10.5^{*}$ & $63.3 \pm 11.8$ \\
\hline & $\mathrm{N}$ & $55.6 \pm 11.2$ & $53.3 \pm 12.5$ & $60.1 \pm 11.8$ & $61.6 \pm 13.5$ & $61.4 \pm 13.2$ & $62.4 \pm 15.2^{*}$ & $61.9 \pm 10.3$ & $60.7 \pm 11.5$ \\
\hline \multirow{2}{*}{$\begin{array}{l}\text { SVR (dynes/ } \\
\text { sec/ } \mathrm{cm}^{5} \text { ) }\end{array}$} & $\mathrm{C}$ & $1,509.0 \pm 333.3$ & $2,138.9 \pm 384.8^{*}$ & $2,101.2 \pm 251.0 *$ & $1,926.9 \pm 453.3^{*}$ & $1,759.4 \pm 385.8^{*}$ & $1,516.8 \pm 451.3$ & $1,388.5 \pm 402.6$ & $1,350.8 \pm 353.6$ \\
\hline & $\mathrm{N}$ & $1,538.8 \pm 385.2$ & $1,836.3 \pm 362.3^{*, \dagger}$ & $1,754.6 \pm 280.8^{*, \dagger}$ & $1,607.2 \pm 295.2^{\dagger}$ & $1,559.5 \pm 272.9^{\dagger}$ & $1,483.6 \pm 296.8$ & $1,421.7 \pm 223.8$ & $1,415.5 \pm 289.8$ \\
\hline
\end{tabular}

Values are expressed as means \pm SD. CI: cardiac index, SV: stroke volume, SVR: systemic vascular resistance, C: control group, N: nitroglycerin group, T1: 10 min after induction of anesthesia, T2: 5 min after $\mathrm{CO}_{2}$ insufflation with supine position, T3: 10 min after the Trendelenburg position with pneumoperitoneum, T4: $30 \mathrm{~min}$ after the Trendelenburgposition with pneumoperitoneum, T5: 1 hr after the Trendelenburg position with pneumoperitoneum, T6: 5 min after desufflation in the supine position, T7: 10 min after desufflation in the supine position, T8: 30 min after desufflation in the supine position. *Indicates $\mathrm{P}<0.05$ compared with $\mathrm{T} 1 .{ }^{\dagger}$ Indicates $\mathrm{P}<0.05$ compared with the same time point as the $\mathrm{C}$ group. ${ }^{\ddagger}$ Indicates $\mathrm{P}<0.05$ compared with $\mathrm{T} 2$. 
had an increase only at T6. SVR was increased from T2-T5 in $\mathrm{C}$ group while it increased in $\mathrm{N}$ group at $\mathrm{T} 2$ and $\mathrm{T} 3$. Increases in SVR were most marked at T2 $(41.7 \%)$ in the C group and in the $\mathrm{N}$ group (19.4\%). When comparing SVR from T2-T5 between groups, SVR in the $\mathrm{N}$ group was significantly lower than in the $\mathrm{C}$ group (Table 3 ).

\section{Discussion}

Hemodynamic alterations during pneumoperitoneum including a decrease of $\mathrm{CO}$, an increase in arterial blood pressure and increases of both systemic and pulmonary vascular resistance are well known; they require accurate information and adequate management due to the complex pathophysiology which can resemble diseases such as chronic heart failure, as described by Stuthers and Cuschieri [6]. Trendelenburg positioning along with pneumoperitoneum. which secures the visual field during the operation, results in more complex hemodynamic alterations. Surgeries of the upper abdomen such as cholecystectomy and gastrectomy are usually performed with reverse Trendelenburg (head-up) positioning, whereas lower abdominal operations such as colon or pelvic surgeries usually needs the Trendelenburg position and requires prolonged operation times and postural changes due to the complex operation characteristics. While there are controversies with respect to hemodynamic alteration during pneumoperitoneum accompanied with the Trendelenburg position, it is generally considered that increases in CVP and pulmonary artery pressure during pneumoperitoneum are aggravated by Trendelenburg positioning [7].

In terms of the effects of pneumoperitoneum on cardiac preload and renal perfusion, Bergman et al. [8] reported that cardiac preload decreases with pneumoperitoneum and a decrease in preload deepens with concomitant nitroglycerin infusion. Further, renal perfusion is not significantly decreased by pneumoperitoneum alone, but it is decreased when infusion of nitroglycerin is also performed. There is also conflicting data reported by Bishara et al. [9] who found that the use of nitroglycerin prevented the decrease of renal perfusion during pneumoperitoneum. But the above mentioned reports were based on animal experiments focused on renal perfusion and were too limited for studying hemodynamic alterations. Therefore, we designed this study to observe the overall hemodynamic changes during both pneumoperitoneum and the Trendelenburg position as well as to seek any favorable effects of continuous infusion of nitroglycerin on hemodynamic changes during LAR which usually needs both pneumoperitoneum and the Trendelenburg position.

Many studies have aimed at reducing the hemodynamic changes during pneumoperitoneum and the Trendelenburg position. Yoon and Jee. [10] reported that a single injection of nicardipine $(30 \mu \mathrm{g} / \mathrm{kg})$ one minute prior to pneumoperitoneum and the Trelendelenburg position for vaginal hysterectomy successfully inhibited a decrease in CI., and Jeun et al. [11] also reported that in laparoscopic gynecologic operations using a single injection of nicardipine $(10 \mu \mathrm{g} / \mathrm{kg})$ plus continuous infusion at $0.5-2.0 \mu \mathrm{g} / \mathrm{kg} / \mathrm{min}$ partially reduced the hemodynamic changes, although the limitations of their study were the hemodynamic index being confined to blood pressure, HR and CI.

Nitroglycerin is mainly used for the treatment and prevention of ischemic heart disease by dilating the coronary artery, systemic or focal veins with an ordinarily low dose which decreases preload and workload of the right ventricle and decreases the pulmonary artery pressure in addition to dilation of the pulmonary vessels. Nitroglycerin dose not induce remarkable changes in SVR at a low dose but induces a decrease in SVR by dilation of arterioles with a high dose [12]. The usual dose of nitroglycerin is $0.5-2 \mu \mathrm{g} / \mathrm{kg} / \mathrm{min}$ while the dose used in this study was a relatively low at $0.5 \mu \mathrm{g} / \mathrm{kg} / \mathrm{min}$, in consideration of the possibility that high dose nitroglycerin may induce severe hypotension in cases of hypovolemic states.

Analysis of the hemodynamic changes in patients demonstrated that MAP increased significantly during pneumoperitomeum at $\mathrm{T} 1$ in both groups with a maximum of $32 \%$. Increases in blood pressure were maintained also during the Trendelenburg position ( $\mathrm{T} 3-\mathrm{T} 5)$, with a $23 \%$ increase in the $\mathrm{N}$ group which was relatively low compared to the $\mathrm{C}$ group at all periods. These results are likely from the vasodilatory effect of nitroglycerin with a corresponding decrease in preload; the increase of blood pressure due to the pneumoperitoneum could be ameliorated in some degree by concomitant use of nitroglycerin. In terms of the HR changes, the $\mathrm{C}$ group showed notable decreases during pneumoperitoneum (T2-T5) which returned to T1 levels after the removal of pneumoperitoneum and the $\mathrm{N}$ group exhibited the least alterations during all periods. The decrease in HR was contrary to previous reports [3] which showed increased HR during pneumoperitoneum and no significant changes in HR during pneumoperitoneum [4]. Remifentanil given during anesthesia was a presumable cause of the bradycardia [13] but the mechanism of no change in HR is not known. The reason of the absence of significant HR decreases in the $\mathrm{N}$ group was presumed to offset the reflex tachycardia of nitroglycerin by the bradycardiac effect of remifentanil.

The CVP during anesthesia increased in both groups, especially in the Trendelenburg position when compared to pneumoperitoneum only (T2). The causes of the elevated CVP were the increase of intra-abdominal pressure (IAP) by pneumoperitoneum and increased venous return by 
the Trendelenburg position which is suppored by the previous report of Horvath et al. [14]. According to Blobner et al. [15], moderate increases in IAP by $12 \mathrm{mmHg}$ during pneumoperitoneum induces the compression of the splanchnic vein leading to an increase in venous return, whereas high increases in IAP (i.e. $>20 \mathrm{mmHg}$ ) causes compression of the inferior vena cava (IVC) resulting in a decrease in venous return. Pneumoperitoneum, which was maintained at 12$13 \mathrm{mmHg}$ in this study generally resulted in an increase in venous return. Andersson et al. [16] advocated that increases in CVP is irrelevant to the amount of venous return by a pneumoperitoneum and it is the Trendelenburg position that affects the increase in CVP most. This was different from our study where the Trendelenburg position was the primary cause of increased CVP.

CVP was maintained but it was not significantly lower in the $\mathrm{N}$ group from $\mathrm{T} 5-\mathrm{T} 7$ compared to the $\mathrm{C}$ group and this was likely due to venous dilatation resulting in a decrease of return volume to the heart. This supports the use of nitroglycerin in relieving the anticipated increase in CVP and consequently the increase of pulmonary artery pressure during pneumoperitoneum and Trelenburg positioning. As a result, this contributed to the hemodynamic stability of patients receiving LAR with high cardiac risk [17].

There are controversies with respect to changes in CO during pneumoperitoneum, but most studies describe through various mechanisms that elevated IAP by pneumoperitoneum results in the reduction of IVC, followed by venous stasis in the lower extremities; this results in reduced venous return to the heart and a decrease in CO [18]. Also, the possibility of increased venous return in the Trendelenburg position, which relieves the decrease of CO, has been suggested [19]. In this study, the transient increase of CI even after Trendelenburg positioning was not observed when compared to the supine position with pneumoperitoneum (T2). CI in this study significantly decreased in both groups at T4-T5 compared to $\mathrm{T} 1$, and relating to the fact that SV significantly increased after a considerable passage of time from induction of pneumoperitoneum and Trendelenburg positioning, the increase in SVR was the most important cause of decreased CI. Hofer et al. [20] claimed that intrathoracic blood volume was the most important factor affecting $\mathrm{CO}$ after pneumoperitoneum and body positioning. They concluded that the change in $\mathrm{CO}$ was very variable and is mostly affected by the increase of intrathoracic pressure from elevated IAP and not by the increased venous return from increased CVP after Trendelenburg positioning and pneumoperitoneum.

Intergroup differences of CI was small despite that the decrease in SVR in the $\mathrm{N}$ group was more prominent than the $\mathrm{C}$ group and we speculate that the reason for a small intergroup difference is due to a smaller SV in the $\mathrm{N}$ group than C group. Sumpelmann et al. [21] claimed that during prolonged pneumoperitoneum of more than 2 hours even at a low pressure of $8 \mathrm{mmHg}$, a decrease of $\mathrm{CI}$, deepening of acidosis, and hypovolemia are difficult to detect with standard routine monitoring. Therefore, invasive monitoring devices for central venous oxygen saturation are needed and as our study that the extensive monitoring of hemodynamic parameters would be necessary for the patient safety. SVR increased by $65 \%$ after pneumoperitoneum and is caused by humoral mediators such as catecholamines, renin-angiotensin system and vasopressin [22]. Vasopressin is proportionally increased with elevated intrathoracic and transmural right atrial pressure [23]. Mechanical stimulation of the intraabdominal receptors also increases vasopressin secretion [24] as well as an increase of blood pressure and SVR. The Trendelenburg position may relieve the increase in SVR whereas inhalation of vasodilative anesthetics like isoflurane and vasodilators such as nitroglycerin and nicardipine can also reduce the increase in SVR [25]. In this study, a $42 \%$ increase in SVR was also noted during pneumoperitoneum in the $\mathrm{C}$ group and SVR decreased to $16 \%$ during Trendelenburg positioning, Further, in the N group, a 19\% increase in SVR during pneumoperitoneum occurred which gradually recovered in the Trendelenburg position and then returned to anesthesia induction (T1) level at T5 point. Considering the significantly lower SVR during all periods after both pneumoperitoneum and Trendelenburg positioning in the $\mathrm{N}$ group compared to the $\mathrm{C}$ group, this study demonstrated the vasodilative effect of nitroglycerin.

In conclusion, this study suggests there were partially successful hemodynamic effects by nitroglycerin infusion including an increase in MAP and SVR during LAR performed under pneumoperitoneum and Trendelenburg positioning. But, this study was restruced because pulmonary artery catheterization for monitoring pulmonary artery and capillary wedge pressure was not monitored and further complementary studies should be performed.

\section{References}

1. Grace PA, Quereshi A, Coleman J, Keane R, McEntee G, Broe P, et al. Reduced postoperative hospitalization after laparoscopic cholecystectomy. Br J Surg 1991; 78: 160-2.

2. Fahy BG, Barnas GM, Flowers JL, Nagle SE, Njoku MJ. The effects of increased abdominal pressure on lung and chest wall mechanics during laparoscopic surgery. Anesth Analg 1995; 81: 744-50.

3. Koivusalo AM, Lindgren L. Effects of carbon dioxide pneumoperitoneum for laparoscopic cholecystectomy. Acta Anaesthesiol Scand 2000; 44: 834-41.

4. Falabella A, Moore-Jeffries E, Sullivan MJ, Nelson R, Lew M. Cardiac function during steep Trenedelenburg position and 
$\mathrm{CO}_{2}$ pneumoperitoneum for robotic-assisted prostatectomy: a transesophageal Doppler probe study. Int J Med Robot 2007; 3: 312-5.

5. Russo A, Marana E, Viviani D, Polidori L, Colicci S, Mettimano M, et al. Diastolic function: the influence of pneumoperitoneum and Tredelenburg positioning during laparoscopic hysterectomy. Eur J Anaesthesiol 2009; 26: 923-7.

6. Struthers AD, Cuschieri A. Cardiovascular consequences of laparoscopic surgery. Lancet 1998; 352: 568-70.

7. Hirvonen EA, Nuutinen LS, Kauko M. Hemodynamic changes due to Trendelenburg positioning and pneumoperitoneum during laparoscopic hysterectomy. Acta Anaesthesiol Scand 1995; 39: 94955.

8. Bergman S, Nutting A, Feldman LS, Vassiliou MC, Andrew CG, Demyttenaere S, et al. Elucidating the relationship between cardiac preload and renal perfusion under pneumoperitoneum. Surg Endosc 2006; 20: 794-800.

9. Bishara B, Karram T, Khatib S, Ramadan R, Schwartz H, Hoffman A, et al. Impact of pneumoperitoneum on renal perfusion and execretory function: beneficial effects of nitroglycerine. Surg Endosc 2009; 23: 568-76.

10. Yoon HJ, Jee YS. Hemodynamic effects of intravenous bolus dosing of nicardipine on pneumoperitoneum during laparoscopicallyassisted vaginal hysterectomy. Korean J Anesthesiol 2006; 50: s43-7.

11. Jeun WJ, Choi YJ, Lee GS, Shim JH, Cho SY. Effect of nicardipine on induction, maintenance and recovery during gynecological laparoscopic surgery. Korean J Anesthesiol 2006; 50: 515-8.

12. Imhof PR, Ott B, Frankhauser P, Chu LC, Hodler J. Difference in nitroglycerin dose-response in the venous and arterial beds. Eur J Clin Pharmacol 1980; 18: 455-60.

13. Watanabe M, Shimizu H, Kikuchi C. Anesthetic management with remifentanil infusion during laparoscopic cholecystectomy. Masui 2009; 58: 1232-5.

14. Horvath KD, Whelan RL, Lier B, Viscomi S, Barry L, Buck K, et al. The effects of elevated intraabdominal pressure, hypercarbia, and positioning on the hemodynamic responses to laparoscopic colectomy in pigs. Surg Endosc 1998; 12: 107-14.

15. Blobner M, Bogdanski R, Kochs E, Henke J, Findeis A, JelenEsselborn S. Effects of iintraabdominal insufflated carbon dioxide and elevated intrabdominal pressure on splanchnic circulation. Anesthesiology 1998; 89: 475-82.

16. Andersson L, Wallin CJ, Sollevi A, Odeberg-Wernerman S. Pneumoperitoneum in healthy humans dose not affect central blood volume or cardiac output. Acta Anaesthesiol Scand 1999; 43: 80914.

17. Odeberg S, Ljungqvist O, Svenberg T, Gannedahl P, Bäckdahl M, von Rosen A, et al. Haemodynamic effects of pneumoperitoneum and the influence of posture during anaesthesia for laparoscopic surgery. Acta Anaesthesiol Scand 1994; 38: 276-83.

18. Goodale RL, Beebe DS, McNevin MP, Boyle M, Letourneau JG, Abrams JH, et al. Hemodynamic, respiratory, and metabolic effects of laparoscopic cholecystectomy. Am J Surg 1993; 166: 533-7.

19. Sharma KC, Brandstetter RD, Brensilver JM, Jung LD. Cardiopulmonary physiology and pathophysiology as a consequence of laparoscopic surgery. Chest 1996; 110: 810-5.

20. Hofer CK, Zalunardo MP, Klaghofer R, Spahr T, Pasch T, Zollinger A. Change in intrathoracic blood volume associated with pneumoperitoneum and positioning. Acta Anaesthesiol Scand 2002; 46: 303-8.

21. Sümpelmann R, Schuerholz T, Marx G, Härtel D, Hecker H, Ure BM, et al. Haemodynamic, acid-base and blood volume change during prolonged low pressure pneumoperitoneum in rabbits. Br J Anaesth 2006; 96: 563-8.

22. Harris SN, Ballantyne GH, Luther MA, Perrino AC Jr. Alteration of cardiovascular performance during laparoscopic colectomy: A combined hemodynamic and echocardiographic analysis. Anesth Analg 1996; 83: 482-7.

23. Solis-Herruzo JA, Moreno D, Gonzalez A, Larrodera L, Castellano G, Gutierrez J, et al. Effects of intrathoracic pressure on plasma arginine vasopressin levels. Gastroenterology 1991; 101: 607-17.

24. Melville RJ, Forsling ML, Frizis HI, LeQuesne LP. Stimulus for vasopressin release during elective intra-abdominal operations. $\mathrm{Br}$ J Surg 1985; 72: 979-82.

25. Feig BW, Berger DH, Dougherty TB, Dupuis JF, His B, Hickey $\mathrm{RC}$, et al. Pharmacologic intervention can reestablish baseline hemodynamic parameters during laparoscopy. Surgery 1994; 116: 733-9; discussion 739-41. 